\title{
COMPORTEMENT DU NITROSYL-RUTHENIUM SANS ENTRAINEUR DANS UNE EAU DE MER SIMULEE
}

\author{
Y. BELOT et M. ARCHAMBEAUD* \\ (manuscrit rę̧u le 18 février 1967 )
}

\begin{abstract}
RÉSUMÉ
Des solutions plus ou moins riches en complexes nitro ont été préparées en traitant une solution de mononitrato nitrosyl-ruthénium sans entraîneur avec des oxydes d'azote. Ces solutions ont été diluées dans des eaux de mer simulées, tamponnées à $p \mathrm{H} 8,4$, la concentration finale en ruthénium étant environ $10^{-9} \mathrm{M}$. L'évolution dans le temps des complexes nitro a été comparée à celle des complexes nitrato en utilisant leurs propriétés d'ultrafiltration et de sorption comme indice de leur état physico-chimique.

Certains complexes nitro restent à l'état soluble et sont très peu retenus par les sorbants argileux, Les complexes nitrato se transforment en espèces colloïdales rapidement adsorbées par toute particule solide en suspension. Ces observations peuvent servir de point de départ à des études ultérieures sur le comportement chimique et radioécologique du nitrosyl-ruthénium marin.
\end{abstract}

\begin{abstract}
Solutions more or less enriched in nitro complexes have been produced by treating a solution of the nitrosyl mononitrate of carrier-free ruthenium 106 with oxides of nitrogen. These solutions have been diluted with simulated seawaters buffered at $p \mathrm{H} \mathrm{8.4} \mathrm{the} \mathrm{final} \mathrm{ruthenium} \mathrm{concentration} \mathrm{being} \mathrm{about} 10^{-9} \mathrm{M}$. The evolution of nitro complexes has been compared with that of nitrato complexes using their ultrafiltration and sorption properties as an indication of their physico-chemical form. Some nitro complexes are soluble and poorly retained by argillaceous sorbents, in contrast to the nitrato complexes producing colloïdal species and being easily adsorbed by any solid suspended matter. These observations may guide further studies on the chemical and radioecological behaviour of the marine nitrosyl-ruthenium.
\end{abstract}

\section{INTRODUCTION}

Le ruthénium 103-106 constitue une partie importante de la radioactivité des effluents rejetés par les usines de traitement des combustibles irradiés dans les eaux douces et marines. Le comportement de ce ruthénium dans l'environ-

* Département de la Protection Sanitaire, Centre d'Etudes Nucléaires de Fontenay-auxRoses, B.P. 6, 92-Fontenay-aux-Roses. 
nement naturel est conditionné par son état physico-chimique, problème fondamental qui doit être abordé avant toute expérimentation radioécologique proprement dite.

\section{L'origine de ce ruthénium est la suivante :}

Lorsque les combustibles nucléaires irradiés sont dissous dans l'acide nitrique en vue de leur traitement chimique, des dérivés de nitrosyl-ruthénium (RuNO) sont formés. Ces dérivés comprennent des complexes nitrato, par exemple $\left[\mathrm{RuNO}\left(\mathrm{NO}_{3}\right)_{3}\left(\mathrm{H}_{2} \mathrm{O}\right)_{2}\right]$ et des complexes nitro tels que [RuNO$\left(\mathrm{NO}_{2}\right)_{2}$ $\left.\left(\mathrm{H}_{2} \mathrm{O}\right)_{2}(\mathrm{OH})\right]$. Ces complexes persistent tout au long des différentes étapes du traitement et apparaissent dans les eaux de lavage, dans les résidus d'extraction par solvant, etc. [6]. Ces solutions résiduaires, avant d'être rejetées, sont partiellement décontaminées par coprécipitation en milieu alcalin. A partir de cette ultime étape, l'évolution des compleses primitifs est assez mal connue. Des chercheurs de l'U.K.A.E.A. ont émis l'hypothèse que les complexes nitrato sans entraîneur pourraient s'hydrolyser en hydroxydes de RuNO, alors que les complexes nitro seraient relativement stables [3]. Ce problème a été abordé ici expérimentalement, dans le cas précis du rejet dans les eaux marines, et en simplifiant au maximum les systèmes chimiques mis en cause, de façon à mettre en lumière les tendances d'un comportement a priori compliqué. Une solution nitrique enrichie en complexes nitro a été préparée. Cette solution a été alcalinisée par dilution dans une eau de mer simulée, tamponnée à $p \mathrm{H} \mathrm{8,4.} \mathrm{L'évolution} \mathrm{dans}$ le temps des complexes nitro a été comparée à celle des complexes nitrato, en soumettant les eaux de mer, ainsi contaminées, à l'ultrafiltration et parallèlement à des essais de sorption sur montmorillonite.

\section{MATÉRIAUX ET MÉTHODES}

\section{II.I. SOLUTIONS-MÈrES DE RUTHÉNIUM 106 :}

Les solutions-mères sont des solutions nitriques contenant des complexes nitrato et des complexes nitro de nitrosyl-ruthénium.

(I) Solution-mère standard : c'est une solution nitrique $\mathrm{M}$, obtenue par dilution de la solution nitrique $8 \mathrm{M}$, fournie par le Radiochemical Centre AMERSHAM. Dans le but d'évaluer les proportions des différents complexes, cette solution a été contrôlée par chromatographie sur papier, selon le procédé recommandé par SCARGILl et Lyon [7]. Le chromatogramme (a) de la figure i permet d'estimer les pourcentages des complexes suivant la nomenclature de FLETCHER et de ses collaborateurs [4] : groupe A (6I \%); groupe B (is \%); groupe C (is \%); complexes dinitro (8\%) [r] [8].

(2) Solution-mère enrichie en complexe nitro: il n'est pas possible de préparer et d'isoler un composé spécifique de nitro nitrosyl-ruthénium sans entraîneur, mais des solutions plus ou moins enrichies en complexes nitro ont été préparées par une méthode inspirée des travaux de GARDNER [6] et BRUCE [2].

Dans $s \mathrm{ml}$ de la solution nitrique $\mathrm{M}$ standard, chauffés à $80^{\circ} \mathrm{C}$, on a fait barboter des oxydes d'azote produits par action d'acide sulfurique $2 \mathrm{~N}$ sur du nitrite de sodium solide. La durée du contact a été de 30 minutes pour une première préparation et de 60 minutes pour une deuxième préparation. Le chromato- 
gramme (b) de la figure I permet d'évaluer la répartition des complexes dans la première préparation : groupe A $(35 \%)$; groupe B ( $11,5 \%)$, groupe $\mathrm{C}(32 \%)$; complexes dinitro $(2 \mathrm{r}, \mathrm{I} \%)$. Il y a eu manifestement augmentation des complexes dinitro, le chromatogramme ne permet pas de distinguer les complexes mononitro des complexes mononitrato.

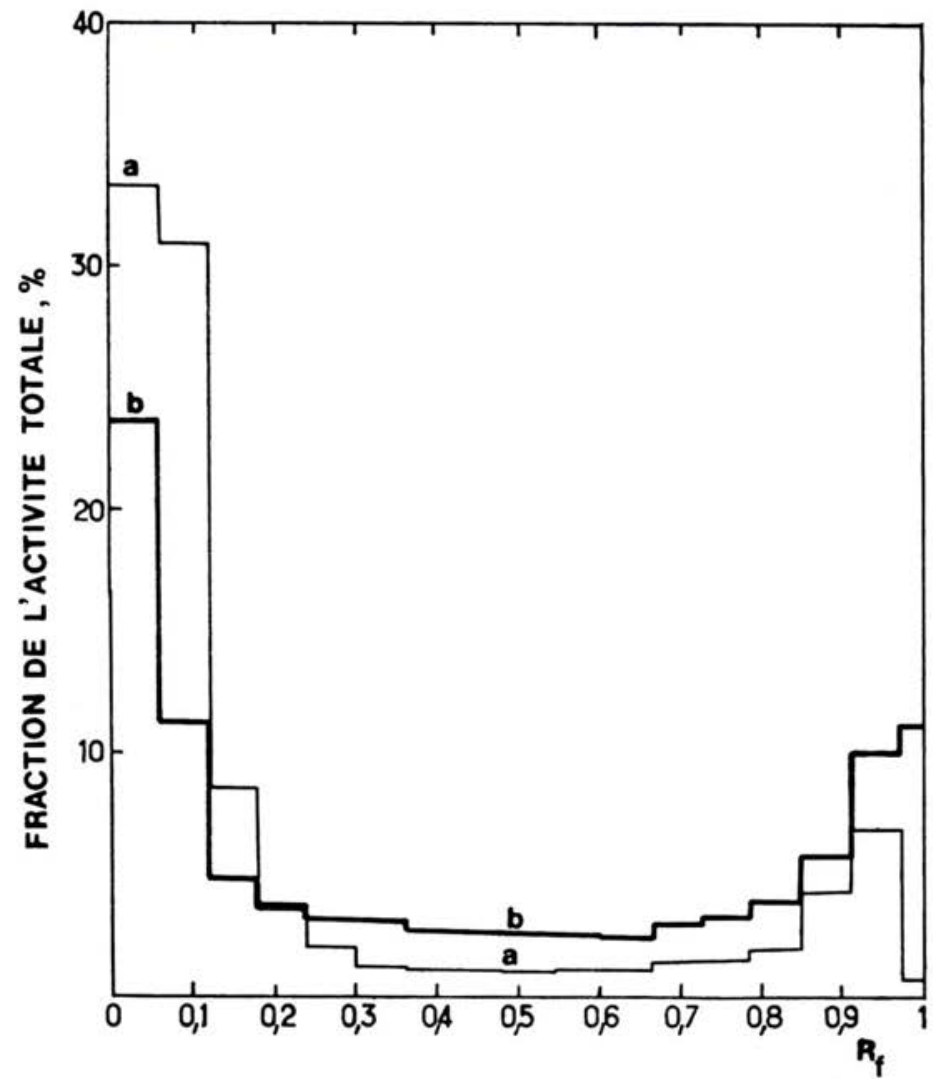

FIG. I. - Chromatogrammes sur papier de composés de nitrosyl-ruthénium

a) solution-mère riche en complexes nitrato (acide nitrique $M$ )

b) solution enrichie en complexes nitro (acide nitrique $\mathrm{M}$ ).

Le solvant utilisé est la méthylisopropylcétone équilibrée avec de l'acide nitrique ${ }_{3} \mathrm{M}$.

\section{iI.2. Préparation des eaux de mer contaminées :}

A partir des solutions-mères précédentes, on a préparé des eaux de mer simulées, contaminées en ruthénium.

Le procédé de préparation est le suivant :

$\mathrm{s} \mathrm{ml}$ de solution-mère sont dilués à un litre de façon à obtenir une solution tamponnée ayant la composition suivante : 0,5 $\mathrm{M}$ en chlorure de sodium-0,005 $\mathrm{M}$ 
en acide borique - $0,005 \mathrm{M}$ en tétraborate de sodium - $0.005 \mathrm{M}$ en acide nitrique (l'acide nitrique étant apporté par la solution-mère).

Les sels utilisés sont des sels de pureté analytique, le $p \mathrm{H}$ obtenu est $8,35 \pm 0.0$ 5. Cette solution tamponnée a le même $p \mathrm{H}$ et la même charge en chlorure de sodium qu'une eau de mer naturelle, elle constitue une eau de mer simulée, contaminée par du ruthénium sans entraîneur.

La solution $\mathrm{A}$ est contaminée à partir de la solution-mère standard. Les solutions $\mathrm{B}$ et $\mathrm{C}$ sont contaminées à partir des solutions-mères enrichies en complexes nitro (première préparation et deuxième préparation).

Les solutions $\mathrm{A}^{\prime}$ et $\mathrm{B}^{\prime}$ ont été obtenues par le procédé suivant : $50 \mathrm{ml}$ de solution contaminée $\mathrm{A}$ (ou B) sont enfermés dans un sac à dialyse, qui est plongé dans $200 \mathrm{ml}$ d'eau de mer simulée inactive, une partie du ruthénium diffuse dans la solution externe qui constitue la solution $\mathrm{A}^{\prime}$ (ou $\mathrm{B}^{\prime}$ ).

\section{II.3. MÉthode D'Ultrafiltration :}

Le procédé suivant a été mis au point, il utilise un matériel standard et permet d'effectuer des filtrations en série de façon simple. Dans un boyau à dialyse en cellulose Visking (diamètre moyen de pores 2,4 millimicrons) sont introduits $5 \mathrm{ml}$ de la solution à filtrer, le boyau noué à ses deux extrémités est placé dans un tube en polyéthylène construit à partir de boîtes à peser standards ( $c f$ : fig. 2). L'ensemble est centrifugé à $2000 \mathrm{tr} / \mathrm{mn}$ pendant une heure.

Des prélèvements de $0,1 \mathrm{ml}$ sont effectués dans la solution primitive et dans le filtrat, pour en mesurer la radioactivité spécifique, le rapport des deux valeurs obtenues constitue le pourcentage de ruthénium ultrafiltrable. Ce pourcentage est mesuré sur quatre fractions successives du filtrat correspondant chacune au volume recueilli pendant une heure de centrifugation. A partir des quatre valeurs ainsi obtenues, on calcule une moyenne et un écart quadratique moyen.

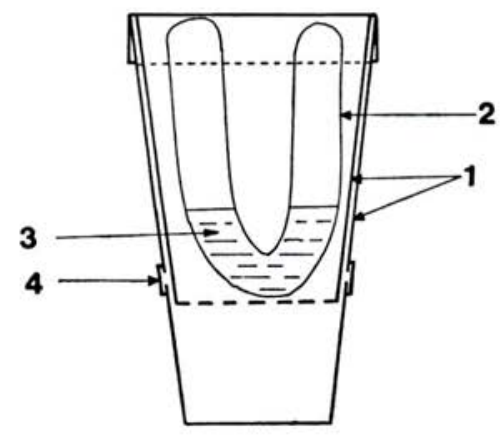

FIG. 2. - Dispositif pour ultrafiltration par centrifugation.

I boîtes à peser de $100 \mathrm{ml}$ en polyéthylène.

2. boyau à dialyse en cellulose Visking.

3. liquide à filtrer.

4. ruban adhésif. 


\section{II.4. MÉthode DE SORPTION :}

Le sorbant choisi est une montmorillonite sodique, minéralogiquement pure, préparée à partir de montmorillonite naturelle brute de Marnia, purifiée par décantation, mise sous forme sodium et lavée par dialyse. Ce matériau a été conservé sous forme de suspension dans de l'eau distillée, à basse température et à l'obscurité pour éviter le développement des algues.

La sorption du ruthénium est étudiée en système clos, de la façon suivante :

Dans un erlen de $250 \mathrm{ml}$, avec agitation magnétique, sont introduits $200 \mathrm{ml}$ d'eau de mer simulée inactive et $5 \mathrm{ml}$ d'eau de mer radioactive. A l'instant zéro de l'expérience, on ajoute la montmorillonite préalablement coagulée. A des temps déterminés, des échantillons homogènes de $2 \mathrm{ml}$ sont prélevés et centrifugés, la radioactivité de la phase liquide est mesurée par comptage $\gamma$ d'échantillons liquides de $\mathrm{I} \mathrm{ml}$. Les courbes tracées donnent le pourcentage de ruthénium non sorbé par rapport au ruthénium initial en fonction du temps. Tous les essais sont réalisés avec des conditions de température et d'agitation identiques.

\section{5. Mesures de RAdioactivité :}

Les concentrations relatives en ruthénium 106 sont déterminées par comptage $\gamma$ du rhodium ro6. Le comptage est effectué sur des échantillons liquides, en utilisant un cristal-puits d'iodure de sodium suivi d'un sélecteur mono-canal. L'erreur statistique est réduite au minimum en comptant s ooo impulsions. L'erreur due à une dérive éventuelle du spectre est corrigée en se référant à un étalon. L'erreur relative globale est évaluée à moins de $3 \%$.

\section{RÉSULTATS}

\section{iII. I. Ultrafiltration :}

Schématiquement le ruthénium ultrafiltrable sera assimilé au ruthénium soluble et le ruthénium non filtrable au ruthénium colloïdal. Les formes ultra filtrables ont un diamètre moyen inférieur au diamètre moyen des pores de la membrane (2,4 millimicrons), il est possible de les assimiler rigoureusement à des formes solubles vraies. Les formes non filtrables contiennent la totalité des formes trop volumineuses pour franchir les pores (colloïdes), mais aussi quelques formes solubles adsorbées sur la membrane par effet des forces de Van der Waals.

Les résultats sont rassemblés dans le tableau I.

(I) Lorsque la solution primitive de RuNO est riche en complexes nitrato, la solution alcaline (eau de mer A) est pauvre en ruthénium soluble ( $25 \%$ environ). La légère augmentation du ruthénium soluble en fonction du temps correspond à l'élimination d'une partie des colloïdes par adhésion sur les parois du récipient, ainsi que le montre la diminution de l'activité spécifique du liquide lorsque la solution vieillit. 
(2) Lorsque la solution primitive a été enrichie en complexes nitro, les solutions alcalines correspondantes (eaux de mer $\mathrm{B}$ et $\mathrm{C}$ ) sont relativement riches en ruthénium soluble ( $55 \%$ pour la solution B et $89 \%$ pour la solution $\mathrm{C}$ plus riche en complexes nitro). Ces deux dernières solutions sont stables dans le temps.

(3) Les dialysats $\mathrm{A}^{\prime}$ et $\mathrm{B}^{\prime}$ contiennent $70 \%$ à $95 \%$ de formes solubles.

\section{TABLEAU I}

PourCentage DE RUTHÉNIUM PASSANT A TRAVERS UNE MEMBRANE DE CELLULOSE PAR ULTRAFILTRATION INFLUENCE DU VIEILLISSEMENT

\begin{tabular}{|c|c|c|c|c|}
\hline $\begin{array}{l}\text { Référence } \\
\text { de la solution }\end{array}$ & $\begin{array}{l}\text { Complexes } \\
\text { prédominants } \\
\text { avant hydrolyse }\end{array}$ & $\begin{array}{c}p \mathrm{H} \\
\pm 0,05\end{array}$ & $\begin{array}{l}\text { Age } \\
\text { de la solution } \\
\text { (jours) }\end{array}$ & $\begin{array}{c}\text { Pourcentage } \\
\text { ultrafiltrable } \\
\pm \text { erreur standard (I) }\end{array}$ \\
\hline Eau de mer A & $\begin{array}{c}\text { nitrato } \\
- \\
-\end{array}$ & $\begin{array}{l}8,25 \\
- \\
-\end{array}$ & $\begin{array}{r}1 \\
15 \\
30 \\
40\end{array}$ & $\begin{array}{l}20,6 \pm 2,0 \\
24,1 \pm 0,8 \\
30,2 \pm 0,6 \\
26,5 \pm 1,4\end{array}$ \\
\hline Eau de mer B & $\begin{array}{c}\text { nitro } \\
-\end{array}$ & 8,15 & $\begin{array}{l}30 \\
40\end{array}$ & $\begin{array}{l}54,5 \pm 5,4 \\
57,3 \pm 1,6\end{array}$ \\
\hline Eau de mer $C$ & $\begin{array}{c}\text { nitro } \\
- \\
-\end{array}$ & $\frac{7,90}{-}$ & $\begin{array}{r}1 \\
15 \\
30 \\
40\end{array}$ & $\begin{array}{l}89,2 \pm 2,7 \\
80,1 \pm 1,6 \\
87,2 \pm 1,9 \\
80,8 \pm 2,6\end{array}$ \\
\hline $\begin{array}{l}\text { Eau de mer } A^{\prime} \\
\text { (dialysat de A) }\end{array}$ & $\begin{array}{c}\text { nitrato } \\
- \\
-\end{array}$ & $\begin{array}{l}8,15 \\
- \\
-\end{array}$ & $\begin{array}{r}1 \\
15 \\
30 \\
40\end{array}$ & $\begin{array}{l}84,4 \pm 0,7 \\
74,2 \pm 3,0 \\
79,5 \pm 6,4 \\
83,2 \pm 0,5\end{array}$ \\
\hline $\begin{array}{l}\text { Eau de mer B' } \\
\text { (dialysat de B) }\end{array}$ & $\begin{array}{c}\text { nitro } \\
- \\
- \\
-\end{array}$ & $\begin{array}{l}- \\
\overline{-}\end{array}$ & $\begin{array}{r}1 \\
15 \\
30 \\
40\end{array}$ & $\begin{array}{l}68,7 \pm 2,8 \\
72,0 \pm 2,2 \\
93,3 \pm 4,6 \\
91,8 \pm 3,1\end{array}$ \\
\hline
\end{tabular}

(I) Moyenne et écart quadratique calculés sur 4 mesures distinctes.

\section{2. SORPTION SUR MONTMORILLONITE :}

La montmorillonite est utilisée ici sous forme coagulée pour éviter tout phénomène parasite de coprécipitation. Les résultats sont donnés dans le tableau II et les figures 3 et 4 . La première colonne du tableau donne, dans un but de comparaison, le pourcentage de ruthénium ultrafiltrable. Les résultats peuvent être analysés de la façon suivante :

(I) Globalement, le ruthénium est d'autant plus sorbable que la solutionmère est plus riche en complexes nitrato ( $c f$. fig. 3).

(2) Le ruthénium colloïdal est adsorbé complètement et très rapidement (pendant la première heure de contact). En effet, le pourcentage de ruthénium 


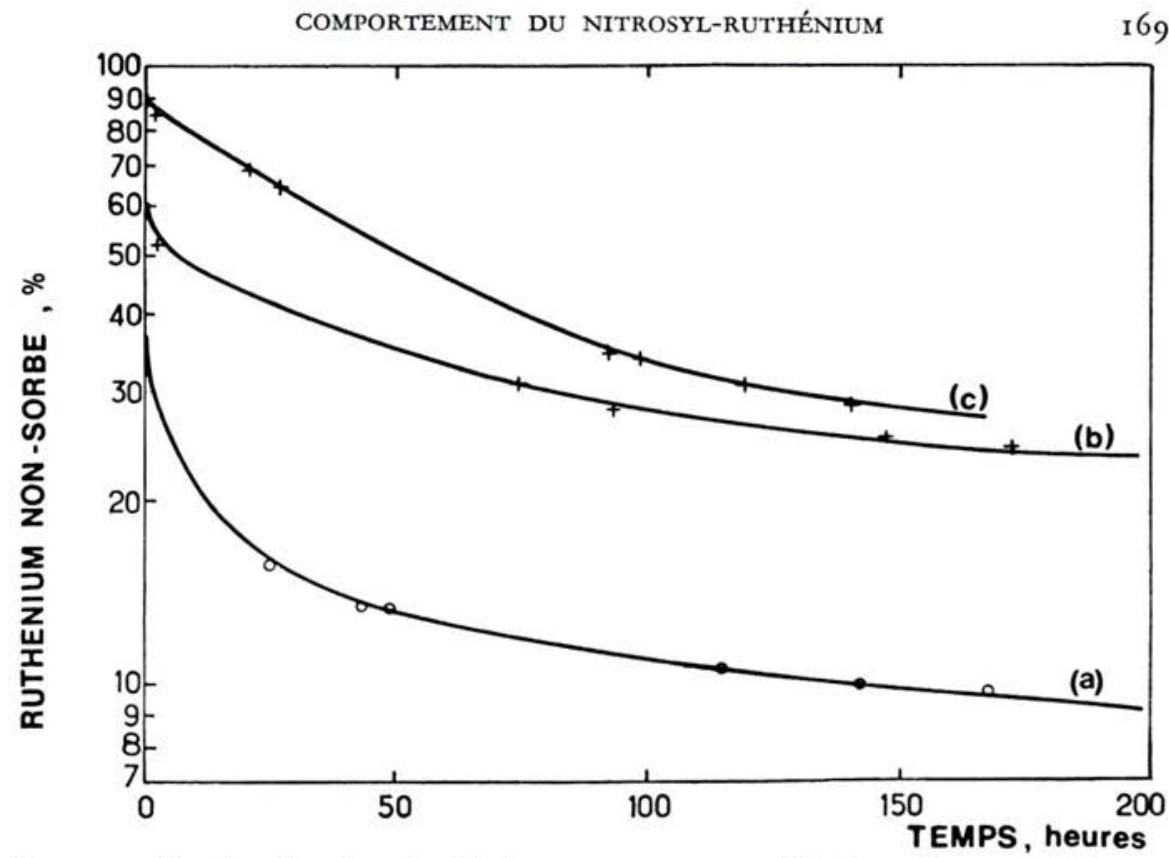

Fig. 3. - Sorption du nitrosyl-ruthénium par une montmorillonite sodique préalablement coagulée (concentration $0,54 \mathrm{~g} .1^{-1}$, température $28^{\circ} \mathrm{C} \pm \mathrm{I}^{\circ} \mathrm{C}$ ).

Solutions : (a) eau de mer A (nitrato); (b) eau de mer B (nitro); (c) eau de mer C (nitro).

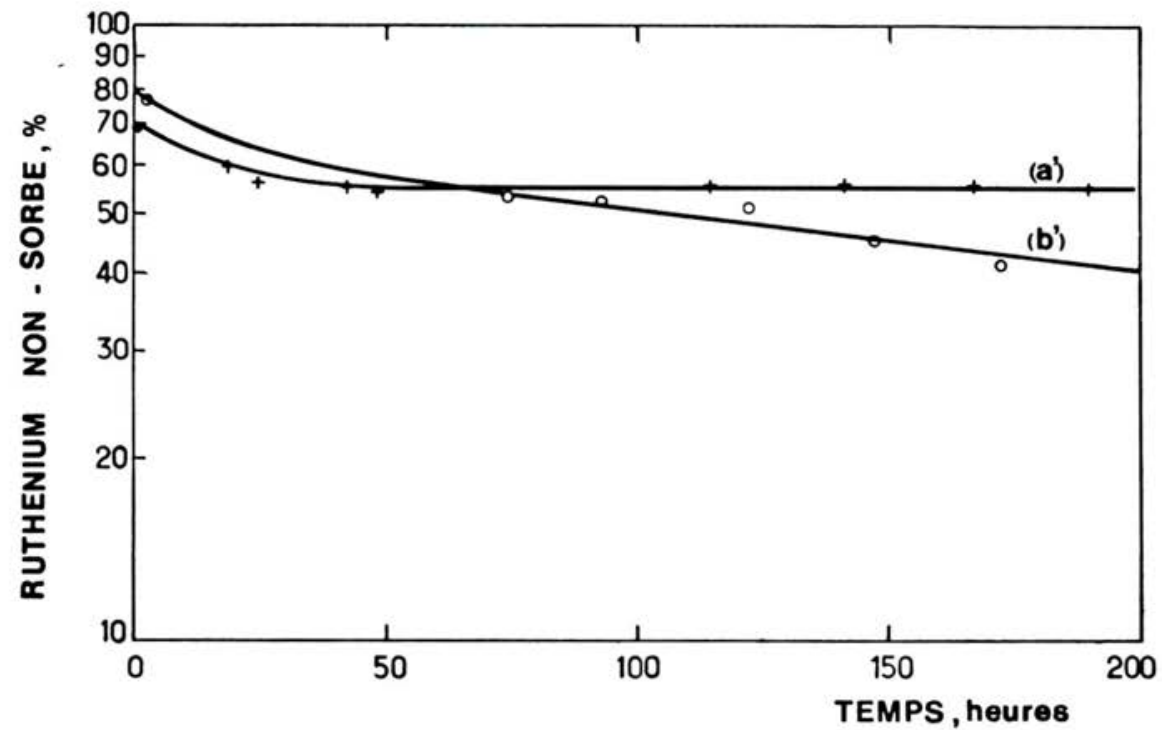

Fig. 4. - Sorption du nitrosyl-ruthénium par une montmorillonite sodique préalablement coagulée (concentration $0,54 \mathrm{~g} .1^{-1}$, température $28^{\circ} \mathrm{C} \pm \mathrm{x}^{\circ} \mathrm{C}$ ).

Solutions : (a') eau de mer $A^{\prime}$ (dialysat de A); (b') eau de mer $B^{\prime}$ (dialysat de B). 
non sorbé, après une heure de contact, est sensiblement égal au pourcentage de ruthénium ultrafiltrable ( $c f$. Tableau II).

(3) Le ruthénium soluble est adsorbé beaucoup plus lentement : il faut en général plusieurs jours de contact pour atteindre un équilibre de sorption apparent. Le mécanisme de cette deuxième étape n'est pas encore complètement élucidé. Cependant tout se passe comme si le ruthénium soluble était un mélange d'espèces lentement sorbables et d'espèces non-sorbables.

(4) Des essais de sorption complémentaires ont été réalisés pour savoir si le ruthénium pouvait entrer en compétition avec le cation magnésium, normalement présent dans les eaux de mer naturelles. Dans ces nouveaux essais, la composition de l'eau de mer simulée a été modifiée en ajoutant 0 ,os mole de chlorure de magnésium par litre et en réajustant le $p \mathrm{H}$ à 8,4 . Les résultats obtenus avec et sans magnésium sont strictement identiques.

TABLEAU II

COMPARAISON DU RUTHÉNIUM ULTRAFILTRABLE ET DU RUTHÉNIUM NON SORBÉ

- sorbant : montmorillonite sodique préalablement coagulée.

- concentration du sorbant : 0,5 g. $1^{-1}$.

- température : $28^{\circ} \mathrm{C} \pm \mathrm{I}^{\circ} \mathrm{C}$.

\begin{tabular}{|c|c|c|c|}
\hline $\begin{array}{c}\text { Référence } \\
\text { de la solution }\end{array}$ & $\begin{array}{l}\text { Complexes } \\
\text { prédominants avant } \\
\text { hydrolyse }\end{array}$ & $\begin{array}{c}\text { Pourcentage de Ru } \\
\text { ultrafiltrable }\end{array}$ & $\begin{array}{c}\text { Pourcentage de } \mathrm{Ru} \\
\text { non sorbé après } \mathrm{I} h \\
\text { de contact }\end{array}$ \\
\hline $\begin{array}{l}\text { Eau de mer A } \\
\text { Eau de mer B } \\
\text { Eau de mer C }\end{array}$ & $\begin{array}{l}\text { nitrato } \\
\text { nitro } \\
\text { nitro }\end{array}$ & $\begin{array}{l}25 \\
54,5 \\
87,7\end{array}$ & $\begin{array}{l}32 \\
57,4 \\
91\end{array}$ \\
\hline $\begin{array}{l}\text { Eau de mer } A^{\prime} \\
\text { (dialysat de } A \text { ) }\end{array}$ & nitrato & 75 & 69 \\
\hline $\begin{array}{l}\text { Eau de mer B' } \\
\text { (dialysat de B) }\end{array}$ & nitro & 72 & 79,3 \\
\hline
\end{tabular}

\section{DISCUSSION}

\section{IV.I. INTERPRÉTATION DES RÉSULTATS :}

Des hypothèses peuvent être formulées sur la nature précise des deux formes de ruthénium observées :

(I) Le ruthénium colloïdal peut être constitué par des particules pseudocolloïdales (colloïdes d'adsorption), le ruthénium étant dans ce cas adsorbé sur des impuretés solides de taille colloïdale accidentellement présentes dans la solution. Il peut être aussi constitué par des hydroxydes de nitrosyl-ruthénium polymérisés, dont le produit de solubilité serait extrêmement bas. (La concentration du ruthénium est de l'ordre de $10^{-9} \mathrm{M}$ ).

Ce ruthénium colloïdal est stable dans le temps, il ne semble pas se resolubiliser même après de longues périodes de vieillissement. 
(2) Le ruthénium soluble est vraisemblablement constitué par des complexes nitro. Les espèces solubles non-sorbables pourraient être des complexes dinitro reconnus particulièrement stables et inertes [3]. Des essais sont en cours pour les identifier sans équivoque.

(3) Les résultats obtenus ici sont à rapprocher des observations antérieures de GARDNER et Brown [6] : lorsque du phosphate ferrique est précipité à $p \mathrm{H}$ I I dans une solution contenant des formes nitro et nitrato, $99 \%$ des complexes nitrato sont enlevés de la solution comparés à $14 \%$ seulement des complexes nitro, qui sont donc responsables des facteurs de décontamination relativement bas obtenus pour le ruthénium dans le traitement des effluents. D'autre part, BRUCE et JACKSON [2] avaient remarqué que le traitement de coprécipitation, pour être efficace, ne devait pas être appliqué directement aux solutions nitriques, mais devait être précédé d'une neutralisation à $p \mathrm{H} 8$, effectuée 24 heures avant le traitement. Le présent travail éclaire ces observations en montrant qu'en milieu alcalin préexistent des colloïdes de ruthénium, formés par hydrolyse rapide des complexes nitrato. Tout traitement de coprécipitation ne fait qu'entraîner ces colloïdes préexistants.

\section{IV.2. REJETS EN MER :}

Le comportement du ruthénium rejeté en mer est donc lié aux proportions relatives de complexes nitrato et de complexes dinitro, qui existent dans les solutions résiduaires avant leur neutralisation. Schématiquement, on aboutit après neutralisation, d'une part, à des hydroxydes de RuNO colloïdaux, d'autre part, à des complexes dinitro en solution vraie. Les premiers sont très vite entraînés par les sédiments, alors que les derniers constituent d'assez bons traceurs qui suivent le mouvement de la masse d'eau et de ses turbulences.

L'eau de mer utilisée dans ce travail simule le $p \mathrm{H}$ et la charge en chlorure de sodium de l'eau naturelle, éléments primordiaux en ce qui concerne l'hydrolyse des complexes et leur transformation éventuelle sous l'action du ligand chlorure. Mais cette simulation ne tient pas compte des composants alcalino-terreux de l'eau naturelle [ $c f$. III.2. (4)]. Elle ne tient pas compte des composants organiques qui, étant donné leur importance, nécessitent un travail expérimental distinct.

\section{CONCLUSIONS}

v.r. L'ensemble des résultats montre l'existence en eau de mer simulée d'un ruthénium colloïdal facilement sorbable et d'un ruthénium soluble peu sorbable, des deux formes étant indépendantes et provenant de complexes originels différents (complexes nitrato et dinitro).

Dans les eaux naturelles, le ruthénium colloïdal évolue rapidement vers une forme granulaire par adsorption sur les particules solides en suspension. Le ruthénium soluble très peu retenu par les sorbants naturels, est apparemment assez stable et il peut être transporté très loin de son point d'émission.

v.2. Il en résulte que le comportement global du ruthénium rejeté en mer est conditionné par la “ qualité » du ruthénium résiduaire (pourcentage des formes 
solubles par exemple). La "qualité » du ruthénium dépend du rapport $\mathrm{HNO}_{3} / \mathrm{HNO}_{2}$ dans les solutions nitriques primitives, mais aussi des procédés utilisés pour traiter les effluents avant rejet.

v.3. Dans le but de connaître de façon plus précise le transfert du nitrosylruthénium dans les différents compartiments du milieu marin, il serait utile de comparer expérimentalement en laboratoire le transfert du ruthénium colloïdal et celui du ruthénium soluble, en utilisant comme sources de pollution des aquariums, les solutions synthétiques utilisées dans ce travail. Parallèlement, une étude complémentaire pourrait être entreprise sur le comportement chimique du nitrosyl-ruthénium en présence des grosses molécules organiques contenues dans l'eau de mer.

\section{REMERCIEMENTS}

Nous remercions MM. Bourrier, Knab et Jamet pour l'aide efficace qu'ils nous ont apportée dans le cadre du contrat entre le C.E.A. et le C.E.R.A.F.E.R., Parc de Tourvoie, 92-Antony.

\section{BIBLIOGRAPHIE}

[1] Brown P.G.M. J. Inorg. Nucl. Chem. 13, 73-83 (1960).

[2] BRuce R.S. and JACxson S. Pbysics in Medicine and Biology 7, 463-471 (1963).

[3] Fletcher J.M., Jenkins I.L., Lever F.M., Martin F.S., Powell A.R. and Todd R. J Inorg. Nucl. Chem. $x, 378$ (1955).

[4] Fletcher J.M., Brown P.G.M., Gardner E.R., Hardy C.J. Wain A.G. and Woodhead J.L. J. Inorg. Nucl. Chem. 12, I54-173 (1959).

[5] Fletcher J.M. and al. J. Inorg. Nucl. Cbem. 27, 161 (1965).

[6] Gardner E.R. and Brown P.G.M. U.K.A.E.A. Document AERE-R-3551 (1960).

[7] Scargill D. and Lyon C.E. U.K.A.E.A. Document AERE-R-4292 (1963).

[8] Wain A.G., Brown P.G.M. and Fletcher J.M. J. Inorg. Nucl. Chem. r2, 346 (1959). 